4. Amores A, Force A, Yan YL, et al. Zebrafish hox clusters and vertebrate genome evolution. Science $1998 ; 282$ : 1711-4.

5. Oulion S, Debiais-Thibaud M, d'Aubenton-Carafa Y, et al. Evolution of Hox gene clusters in Gnathostomes: insights from a survey of a shark (Scyliorhinus canicula) transcriptome. Mol Biol Evol $2010 ; 27$ : 2829-38.

6. Ravi V, Lam K, Tay BH, et al. Elephant shark (Callorhinchus milii) provides insights into the evolution of Hox gene clusters in gnathostomes. Proc Natl Acad Sci USA 2009 ; 106 : 16327-32.

7. King BL, Gillis JA, Carlisle HR, Dahn RD. A natural deletion of the HoxC cluster in elasmobranch fishes. Science 2011 ; $334: 1517$.

8. Oulion S, Borday-Birraux V, Debiais-Thibaud M, et al. Evolution of repeated structures along the body axis of jawed vertebrates, insights from the Scyliorhinus canicula Hox code. Evol Dev 2011 ; 13 : 247-59.
9. Kardong KV. Vertebrates: comparative anatomy, function, evolution. Boston: McGraw-Hill Higher Education, 2006 : $782 \mathrm{p}$.

10. McIntyre DC, Rakshit S, Yallowitz AR, et al. Hox patterning of the vertebrate rib cage. Development 2007 ; $134: 2981-9$

11. Wellik DM. Hox genes and vertebrate axial pattern. Curr Top Dev Biol $2009 ; 88$ : 257-78.

\title{
NOUVELLE
}

\section{Approche optogénétique de suppression de la plasticité synaptique induite par la cocaine}

\section{Normalisation de la sensibilisation locomotrice}

Christian Lüscher ${ }^{1,2}$, Vincent Pascoli ${ }^{1}$

\author{
${ }^{1}$ Department of basic neurosciences, medical faculty, \\ university of Geneva, 1211 Geneva, Suisse ; \\ ${ }^{2}$ Clinic of neurology, department of clinical neurosciences, \\ Geneva university hospital, 1211 Geneva, Suisse. \\ vincent.pascoli@unige.ch
}

\section{La plasticité synaptique, support de l'addiction}

La consommation de cocaine provoque des altérations comportementales pouvant conduire à l'addiction. L'addiction est caractérisée par une perte de la liberté individuelle se manifestant par un affaiblissement du contrôle de la consommation en dépit des conséquences néfastes ressenties, et par un fort risque de rechute après une période de sevrage. Une meilleure connaissance des circuits neuronaux impliqués et la modélisation animale des différentes facettes de l'addiction ont permis d'accroître notre compréhension des mécanismes de l'addiction aux drogues. II est notamment établi que les drogues d'abus modifient l'efficacité des connexions entre les neurones dans les circuits cérébraux de la récompense [1] et que cela contribue aux altérations comportementales caractéristiques de l'addiction [2]. La découverte que ces changements (plasticité synaptique) sont réversibles chez la souris établit la preuve de concept que ce type de stratégie pourrait être élaboré pour traiter l'addiction aux drogues [3]. La plasticité synaptique, le processus par lequel les connexions (synapses) entre les cellules nerveuses sont renforcées ou réduites selon leur niveau d'activité, est naturellement essentielle pour le développement et l'apprentissage. Cependant, la plasticité synaptique est également impliquée dans certaines pathologies neurologiques (ou psychiatriques), notamment celles qui résultent de la consommation abusive de drogues, celle-ci représentant alors une forme d'apprentissage pathologique.

\section{Plasticité synaptique induite} par la cocaïne : une caractéristique des neurones MSN exprimant DIR

Nous avons restreint notre étude à une partie du système de récompense, le striatum ventral (ou noyau accumbens [NAc]) et au principal type cellulaire qui le constitue, les neurones épineux de taille moyenne (medium-sized spiny neurons $[\mathrm{MSN}])$. Ces neurones intègrent les signaux provenant des aires limbiques et corticales contrôlant les comportements motivés via le neurotransmetteur glutamate et les signaux transmis par la dopamine et provenant de l'aire tegmentale ventrale (ATV) (Figure I). Puis, ces neurones MSN communiquent avec les circuits moteurs qui engagent la réponse comportementale. La cocaïne, comme les autres drogues addictives, provoque une forte élévation de la concentration en dopamine dans les structures cérébrales recevant les terminaisons des neurones dopaminergiques de l'ATV, notamment le NAc. Cette élévation de dopamine est à l'origine des effets renforçants [4] et d'adaptations neuronales précoces [5]. Les neurones MSN peuvent être divisés en deux populations de même taille selon le sous-type de récepteur de la dopamine qu'ils expriment, DIR ou D2R [6]. L'utilisation de souris transgéniques exprimant la protéine fluorescente eGFP (enhanced green fluorescent protein) spécifiquement dans les MSN de type DIR ou D2R permet l'identification de ces neurones lors des enregistrements électrophysiologiques. Ces deux populations projettent vers différentes structures et ont différentes fonctions, même si ces distinctions sont moins claires pour le NAc que pour le 


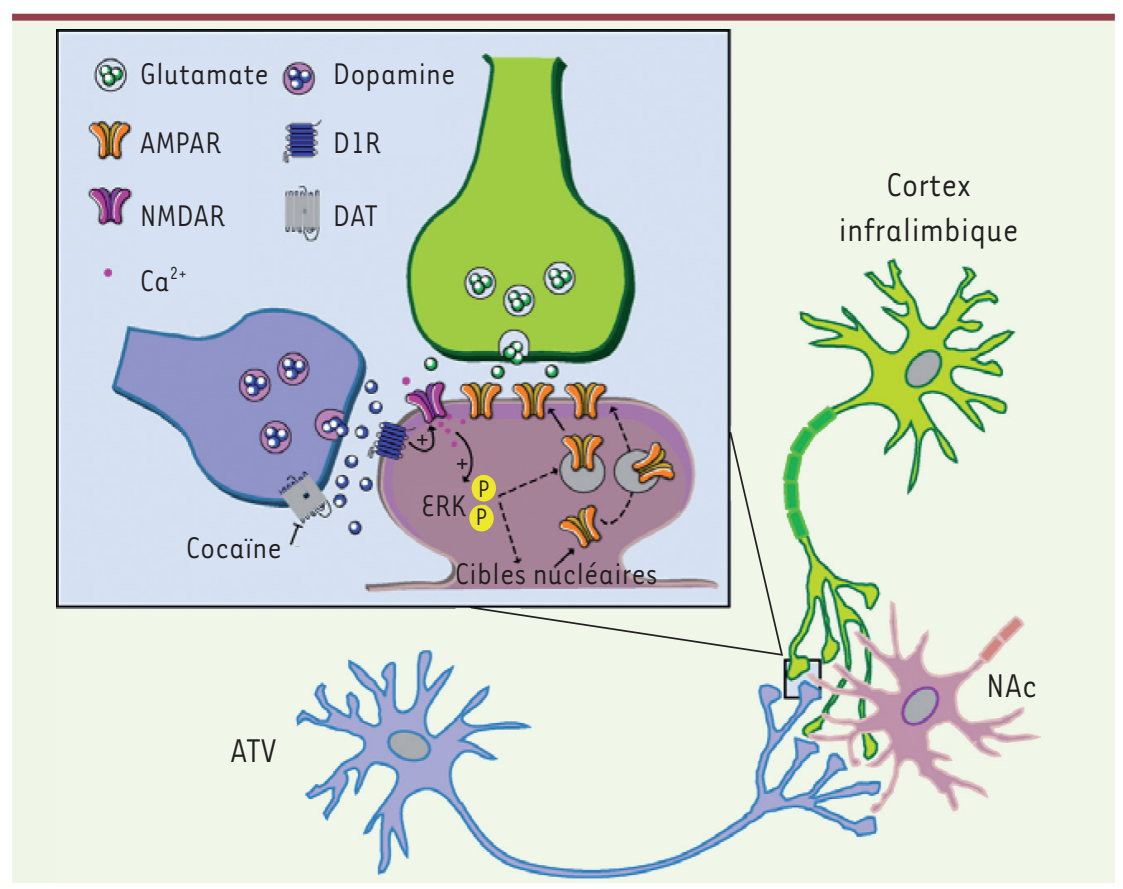

Figure 1. Implication de ERK dans la potentialisation des synapses glutamatergiques induite par la cocaïne. Les neurones (MSN) du noyau accumbens (NAc) reçoivent les terminaisons dopaminergiques provenant de l'aire tegmentale ventrale (ATV) et glutamatergiques du cortex préfrontal (entre autres), pour former des synapses tripartites. La cocaïne provoque une forte augmentation de dopamine (DA) extracellulaire, due à l'inhibition du transporteur de la dopamine (DAT) situé sur les terminaisons des neurones de l'ATV. La stimulation des récepteurs de la dopamine de type Dl (DIR) favorise l'entrée de calcium dans les MSN du NAc via la potentialisation des récepteurs au glutamate de type NMDAR. Cela conduit à l'activation par phosphorylation de la voie ERK qui est impliquée dans la potentialisation synaptique, soit par promotion de l'insertion de récepteurs AMPAR à partir d'un pool intracellulaire, soit en favorisant leur synthèse par une action au niveau transcriptionnel.

striatum dorsal. Nous avons montré que la cocaïne provoquait un renforcement (potentialisation) des synapses glutamatergiques spécifiquement sur les DIR-MSN [3].

\section{Voie de signalisation $\varepsilon$ RK et plasticité synaptique}

Parmi les voies de signalisation engagées par l'excès de dopamine, la phosphorylation de ERK (extracellular signal-regulated kinase) dans le NAc est particulièrement intéressante parce qu'elle est impliquée dans les adaptations comportementales induites par la cocaïne [7] et parce qu'elle est dépendante de la stimulation coordonnée des DIR et des récepteurs au glutamate NMDA $[8,9]$. In vitro, la potentialisation à long terme (LTP) des synapses glutamatergiques
La suppression de la plasticité synaptique induite par la cocaïne efface la sensibilisation locomotrice Nous avons étudié la plasticité synaptique induite par la cocaïne associée à la sensibilisation locomotrice : on désigne ainsi l'augmentation de la réponse locomotrice qui est observée lors d'administrations répétées de la drogue, et qui persiste longtemps après son arrêt. La potentialisation de ces synapses par la cocaïne suit le même décours temporel que la sensibilisation locomotrice : les deux phénomènes sont observés une semaine après l'instauration du traitement à la cocaïne, mais ne le sont plus un mois après. Une théorie influente dans le domaine de l'addiction propose que les propriétés «motivationnelles » de la cocaïne (qui poussent les consommateurs à rechercher la drogue) seraient responsables de la sensibilisation [14]. Pour établir une relation causale entre le renforcement des synapses glutamatergiques chez les animaux sensibilisés à la cocaïne et l'expression comportementale de cette sensibilisation locomotrice, nous avons testé si, en l'absence de potentialisation, la sensibilisation persistait ou était supprimée. Pour cela nous avons utilisé l'optogénétique qui permet de contrôler par des flashs de lumière $(470 \mathrm{~nm})$ l'activité des neurones. En pratique, la protéine sensible à la lumière (channel rhodopsin [ChR2]) est introduite via un vecteur viral dans le cortex infralimbique, et des canules dans lesquelles passent les fibres optiques sont implantées dans le NAc, ce qui permet de stimuler sélectivement par la lumière les terminaisons glutamatergiques. La stimulation de ces neurones à une basse fréquence ( $1 \mathrm{~Hz}$, protocole de long term depression [LTD]) permet la « dépotentialisation » de la transmission glutamatergique sur les MSN par un mécanisme postsynaptique (réduction du nombre de AMPAR à la membrane des MSN). L'application de ce traitement abolit complètement la sensibilisation loco- 


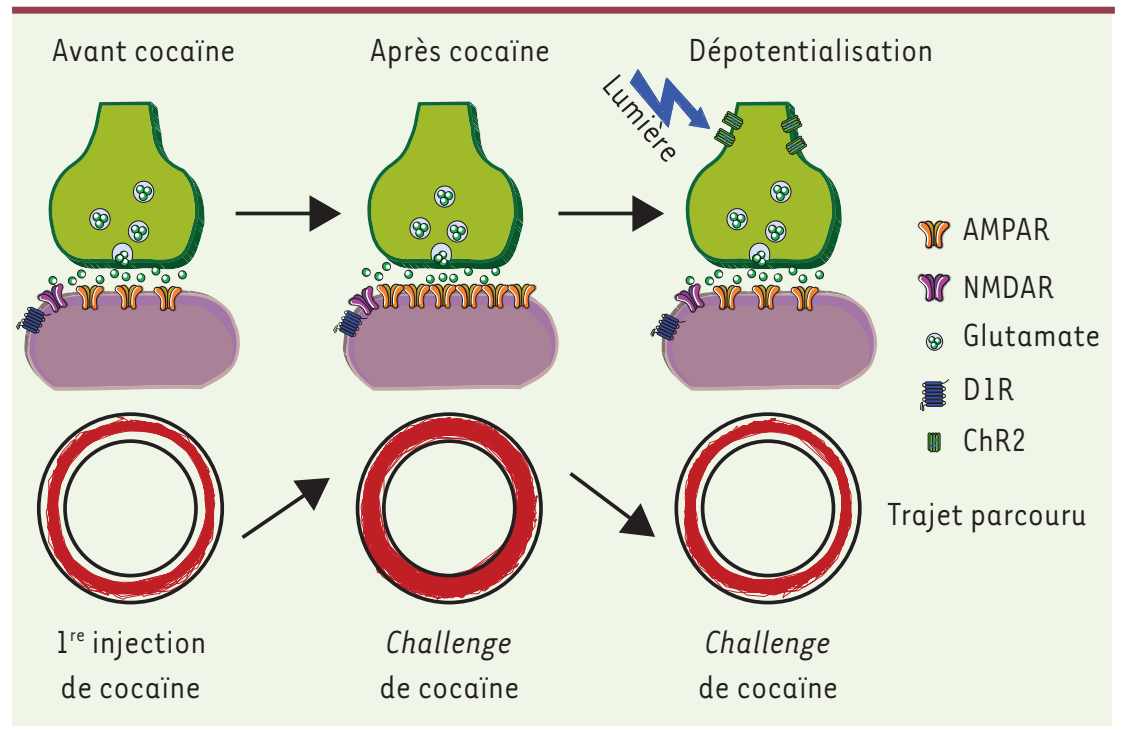

Figure 2. Plasticité synaptique et sensibilisation locomotrice induites par la cocaïne. Les synapses entre les projections du cortex infralimbique et les DIR-MSN du NAc possèdent un pool défini de récepteurs AMPA qui détermine l'efficacité basale de la transmission glutamatergique. L'injection de cocaïne provoque une hyperactivité locomotrice. Après exposition à la cocaïne (une semaine après une injection, ou 10 jours après plusieurs injections), la transmission glutamatergique est renforcée : les synapses sont potentialisées par un mécanisme postsynaptique (insertion de AMPAR). Si on administre à nouveau de la cocaïne à ce stade (challenge), une réponse locomotrice est déclenchée, environ deux fois plus importante que celle qui est observée lors de la première exposition. La réponse est dite sensibilisée. L'application d'un traitement optogénétique (stimulation des afférences glutamatergiques exprimant le channel rhodopsin, à $1 \mathrm{~Hz}$ pendant $10 \mathrm{~min}$, flèche bleue) permet le rétablissement d'une transmission basale par dépotentialisation (réduction des AMPAR membranaires). L'administration de cocaïne (challenge) n'entraîne alors pas de réponse locomotrice sensibilisée. L'activité locomotrice est mesurée dans un couloir circulaire et quantifiée automatiquement par un logiciel dédié. Elle est proportionnelle à l'épaisseur du tracé (ici dans les cercles marrons) (adapté de [16]).

motrice, ne laissant apparaître qu'une réponse non sensibilisée lors d'une réexposition à la cocaïne (Figure 2). Ainsi, en effaçant la plasticité synaptique induite par la cocaïne chez des souris, nous avons rétabli la réponse comportementale initiale [3].

\section{Implications et perspectives}

Cette étude prouve que des neuroadaptations précoces induites par quelques injections de cocaïne sont réversibles. Ceci est surprenant sachant que les individus ayant une addiction ont des changements très persistants de leur cerveau et une vulnérabilité à la rechute tout au long de leur vie. Notre approche est innovante parce qu'elle diffère significativement des approches phar-
Ce traitement par la lumière, en agistion relayée par les mêmes mécanismes cerveau pour réguler l'efficacité de ses ('hez l'homme, cela pourrait tion [DBS]) ou par stimulation magnétique transcrânienne plutôt que par une approche optogénétique [15], mais à ce jour nous n'avons pas de preuve que cette approche soit efficace chez des toxicomanes. Préalablement aux études cliniques chez l'homme, il faudrait évaluer cette stratégie dans des modèles plus sophistiqués d'addiction chez l'ani- mal, impliquant notamment une prise volontaire de cocaïne par l'animal. $\Delta$ Optogenetic reversal of cocaineevoked synaptic potentiation normalizes behavioural sensitization

\section{CONFLITS D'INTÉRÊTS}

Les auteurs déclarent n'avoir aucun conflit d'intérêts concernant les données publiées dans cet article.

\section{RÉFÉRENCES}

1. Lüscher C, Malenka RC. Drug-evoked synaptic plasticity in addiction: from molecular changes to circuit remodeling. Neuron $2011 ; 69: 650-63$.

2. Wolf $M E$, Ferrario CR. AMPA receptor plasticity in the nucleus accumbens after repeated exposure to cocaine. Neurosci Biobehav Rev 2010 ; 35 : 185-211.

3. Pascoli V, Turiault M, Lüscher C. Reversal of cocaineevoked synaptic potentiation resets drug-induced adaptive behaviour. Nature 2011 ; 481 : 71-5.

4. Witten IB, Steinberg દદ, Lee Sy, et al. Recombinasedriver rat lines: tools, techniques, and optogenetic application to dopamine-mediated reinforcement. Neuron $2011 ; 72: 721-33$.

5. Brown MTC, Bellone C, Mameli M, et al. Drug-driven AMPA receptor redistribution mimicked by selective dopamine neuron stimulation. PLoS One $2010 ; 5$ : el5870.

6. Valjent $\varepsilon$, Bertran-Gonzalez J, Hervé D, et al. Looking BAC at striatal signaling: cell-specific analysis in new transgenic mice. Trends Neurosci 2009 ; 32 : 538-47.

7. Girault JA, Valjent $\varepsilon$, Caboche J, Hervé D. ERK2: a logical AND gate critical for drug-induced plasticity? Curr Opin Pharmacol 2007 ; $7: 77-85$.

8. Pascoli V, Besnard A, Hervé D, et al. Cyclic adenosine monophosphate-independent tyrosine phosphorylation of NR2B mediates cocaine-induced extracellular signal-regulated kinase activation. Biol Psychiatry 2011;69:218-27.

9. Valjent $\varepsilon$, Hervé $D$, Girault JA. Drogues addictives, protéine phosphatases et voie ERK. Med Sci (Paris) $2005 ; 21: 453-4$.

10. Shen W, Flajolet M, Greengard P, Surmeier DJ. Dichotomous dopaminergic control of striatal synaptic plasticity. Science 2008 ; 321 : 848-51.

11. Zhu JJ, Qin Y, Zhao M, et al. Ras and Rap control AMPA receptor trafficking during synaptic plasticity. Cell $2002 ; 110: 443-55$.

12. Hervé D. La drogue : une histoire d'histone? Med Sci (Paris) $2010 ; 26: 568-71$.

13. Hervé D, Matamales M, Stipanovich A, et al. Un nouveau mécanisme par lequel la récompense et les drogues modifient la chromatine dans les neurones. Med Sci (Paris) 2008; 24 : 1027-9.

14. Robinson TE, Berridge KC. The incentive sensitization theory of addiction: some current issues. Philos Trans $R$ Soc Lond B Biol Sci 2008 ; 363 : 3137-46.

15. Feil J, Zangen A. Brain stimulation in the study and treatment of addiction. Neurosci Biobehav Rev 2010 ; $34: 559-74$.

16. Wolf ME. Neuroscience: behavioural effects of cocaine reversed. Nature $2012 ; 481: 36-7$.

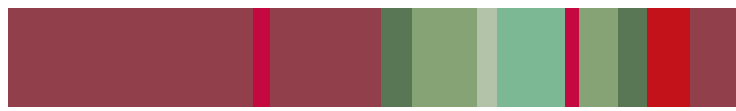

